\title{
A NEW TECHNIQUE FOR BRONCHOGRAPHY IN CHILDREN
}

BY

\author{
JOHN B. BROOKS and JOSEPH W. LEWIS \\ From Harefield Hospital, Harefield, Middlesex
}

(RECEIVED FOR PUBLICATION AUGUST 5, 1955)

Bronchography in children has its own particular problems. Because the diameters of the bronchi are smaller than those in adults the viscosity factor of the contrast medium is relatively more important. The use of a too viscous medium in outlining the bronchial tree may lead to respiratory embarrassment. Bronchial secretion in bronchitic and bronchiectatic cases is also more likely to interfere with the obtaining of a good picture. It is, therefore, important that proper postural drainage be carried out beforehand.

Any method employed must be almost or entirely painless and must, if necessary, be independent of the child's cooperation. It might be thought that bronchography in an anaesthetized child would yield the best results. However, experience with several techniques has shown us that failure to obtain satisfactory bronchograms is frequently due to alveolar flooding. This may be due to hyperpnoea or to controlled or assisted respiration using relaxants.

After using other methods over a considerable period with variable success, we have evolved the method described below. It has been used successfully in $\mathbf{4 0}$ cases of which more than half had gross bronchiectasis and considerable bronchial secretion. These cases are the ones in which really satisfactory bronchograms are difficult to obtain. Our particular aim was not to exclude the presence of gross bronchiectasis (which is easily achieved by almost any method), but to get complete and optimal filling of the whole bronchial tree, resulting in bronchograms good enough for decision for or against operation. The age range of the cases dealt with was from 3 to 17 years, but most were between 4 and 10 .

\section{Materials}

The following are required:

(1) Anaesthetic apparatus for the administration of nitrous oxide and oxygen.

(2) A 10-ml. syringe containing $10 \mathrm{ml}$. of $5 \%$ thiopentone.
(3) A 2-ml. syringe fitted with a No. 12 needle containing $2 \mathrm{ml}$. of $4 \%$ 'xylocaine' (lignocaine hydrochloride) for crico-thyroid or tracheal injection.

(4) A spray containing $4 \mathrm{ml}$. of $4 \%$ 'xylocaine'.

(5) A 2-ml. syringe containing $2 \mathrm{ml}$. of 'scoline' (succinyldicholine chloride) for occasional use.

(6) A No. 1 Magill nasal tube with a small safety-pin stuck through it so that when introduced into the nose as far as the pin its tip is midway between the cords and carina. The pin is also useful in helping to secure the tube to the face with strapping.

(7) A 2-ft. length of No. 4 polythene tubing marked with a small piece of strapping so that when threaded through the Magill tube it projects a quarter to half an inch beyond its end.

(8) Suction apparatus and No. 8 gum elastic catheter for aspiration as necessary through the Magill tube.

(9) A bronchoscopy trolley and child's laryngoscope available for immediate use.

(10) A 20-ml. syringe containing the contrast medium and fitted with a thick needle of suitable diameter to fit tightly into the polythene tubing.

\section{Preliminary Treatment}

Clinical examination will indicate the necessity or otherwise for breathing exercises. If there is secretion, as is frequently the case with bronchitis and bronchiectasis, postural drainage should be carried out regularly. It is particularly important to carry out posturing an hour or two before the actual bronchography as this may make all the difference between a good and a bad bronchogram. In cases where there is much sputum, the responsible organism should be tested for sensitivity and treatment by the appropriate antibiotic instituted. In this connexion we have frequently encountered cases which have been ineffectually treated previously with small doses of antibiotics. A further 10-day course entailing large doses of the order of 1 mega unit twice daily when crystalline penicillin is used had led to marked improvement in many of these cases. When there is no sputum we nevertheless cover the actual bronchography with a five-day routine course of penicillin, since we have found that it reduces the likelihood of systemic disturbance after bronchography. The child should have no food or drink on the morning of bronchography, and it is therefore better for the investigation to be carried out 
early in the day to avoid unnecessary distress. The premedication was 'omnopon' (grain 1/24 per stone body weight) with hyperduric atropine, grain 1/100. Administration of this at the correct time is important. If the child is old enough and intelligent we usually explain beforehand what we are about to do, as we find that we get better cooperation at the time of the bronchography.

\section{Anaesthesia and Procedure}

The child is anaesthetized with a sleeping dose of thiopentone of the order of $0.1 \mathrm{~g}$. in a 4-year-old, and $0.2 \mathrm{~g}$. in a 7-year-old. If an injection is resented, anaesthesia is induced with nitrous oxide and oxygen and the thiopentone given when the child is asleep.

Two millilitres of $4 \%$ 'xylocaine' is now injected either through the crico-thyroid membrane or the trachea itself; the child coughs and spreads the local anaesthetic widely in the trachea and over the larynx. The anaesthetic facepiece is now applied and a mixture of nitrous oxide, oxygen and carbon dioxide given until the child is hyperventilating. While the child inspires deeply, $4 \%$ 'xylocaine' is sprayed up the nostril through which it is proposed to pass the Magill tube. This results in good anaesthesia of the nose and pharynx. The facepiece is reapplied and the same mixture of gas is given. The Magill tube is then passed blindly as the child hyperventilates. In cases of difficulty, the tube can be passed under direct vision, following a small dose of 'scoline'.

At this stage, if secretion is audible through the Magill tube, the gum elastic catheter is pushed down it and the trachea and bronchi aspirated. The catheter is removed, the polythene tube is passed down the Magill tube as far as its mark, and $2 \mathrm{ml}$. of 'xylocaine' injected through it into the lower end of the trachea. The polythene tube is removed and with suitable posturing the solution anaesthetizes the bronchial tree on the side under investigation. Following this, aspiration of secretion or excess anaesthetic solution through the gum elastic catheter is sometimes needed.

In a few minutes the child wakes and as he feels no discomfort with the Magill tube in his nose he is easily reassured. He is taken into the $x$-ray room and the polythene tube is connected to the syringe containing the contrast medium and filled to its end. It is then pushed down the Magill tube as far as its mark. The child is postured to outline each bronchus in turn. For children below the age of 14 we use from 8 to $14 \mathrm{ml}$. of oily 'dionosil' $60 \% \mathrm{w} / \mathrm{v}$, and this is distributed equally throughout the lobes. A proportion of the 'dionosil' is injected for each change of posture. The bronchi are filled in the following order: the basals, the dorsal, the middle on the right, and lastly, the upper lobe bronchi including the lingula if the left lung is being investigated.

The polythene tube is then removed and the radiograph taken. The whole procedure can be done without haste, an important factor in obtaining good quality bronchograms. The child is not connected to the anaesthetic machine and breathes quietly through and around the Magill tube. He is able to hold his breath as directed whilst the radiograph is taken. The child lies quietly while the films are developed and further opaque medium can be injected to outline any segments shown to be inadequately filled. All that is needed is replacement of the polythene tube-a simple matter.

While the best bronchograms are obtained when the patient is conscious and cooperative, the method can still be applied to the sleeping child. Occasionally the child on waking indicates in no uncertain fashion that he does not intend to cooperate. In these circumstances it is desirable to keep him asleep with a further dose of thiopentone. The local anaesthetic prevents bronchial irritation and allows for a light sleep and natural respirations which help to ensure a good bronchogram.

When adequate bronchograms have been obtained the medium is aspirated through the No. 8 gum elastic catheter via the Magill tube. These are then both removed. This action usually provokes coughing and causes the child to bring up further amounts of 'dionosil'. The child is sent back to the ward with the examined lung uppermost. The whole procedure usually takes from 15 to 20 minutes. Nothing is allowed by mouth for two hours until the local anaesthetic has worn off. Postural drainage is continued in the ward until the bronchi are clear.

\section{Discussion}

It will be seen that part of the success of this method is dependent on adequate preparation of the patient along the lines indicated. This has meant that routine bronchoscopy and suction before bronchography has not been necessary and thus this stimulation to further secretion and spasm has been avoided. Secretion is usually satisfactorily removed by a catheter through the Magill tube, and only in two or three cases has it been necessary to carry out bronchoscopy before passing the Magill tube.

Oily 'dionosil', $60 \% \mathrm{w} / \mathrm{v}$, was chosen because it gives radiographs of sufficient contrast; in addition, it does not flow out into the alveoli rapidly and also is absorbed quite quickly, usually in about $\mathbf{7 2}$ hours. We have experienced few side-effects from it and it is much less irritating than the aqueous preparation. Failure to shake the solution adequately did lead to bronchograms of poor contrast on one or two occasions. We had the impression that the contrast factor varied with the particular batch of the preparation.

The anaesthesia involves what may appear to be rather large quantities of 'xylocaine', namely, up to $8 \mathrm{ml}$. in small children and more in the bigger ones. In practice there have been no ill-effects. The amount used is about $0 \cdot 13 \mathrm{~g}$. per stone body weight and probably not all this is absorbed. On a weight basis this is equivalent to the amount used in complete safety in over 300 local anaesthetics for thoracoplasty in adults by one of us.

If the recovery of the patient from the anaesthetic for intubation is delayed then it is important to 
decide whether to prolong it with additional thiopentone or to await complete return to consciousness. Attempts to carry out bronchography at this stage are invariably marred by the child's uncooperativeness as he returns to consciousness. One of the minor disadvantages of the method in our early cases lay in the difficulty of estimating accurately the minimal amount of thiopentone required. Delayed recovery from the intubation anaesthetic may lead to a considerable hold up in the $x$-ray department, a point of some practical importance. With increasing experience this disadvantage was largely eliminated.

The fact that the patient is no longer connected to the anaesthetic machine during the actual bronchography greatly facilitates the filling of the various lobes. His respiration is quiet and natural, and cooperation is good during posturing since he is not apprehensive. While the radiographs are taken the child will usually cooperate by holding his breath as directed. At the stage of unconsciousness at which we do the bronchogram we have been interested to note that little is retained in the child's memory, a point of considerable practical importance when the same procedure may have to be carried out on the opposite lung a week later. The child leaves the $x$-ray department conscious and this makes transport back to the ward and also nursing care much easier.
About a quarter of our cases required extra thiopentone to keep them asleep. The respirations are quiet and natural. The disadvantages are that the radiographer is without the child's cooperation and also that the final films are not of such good quality as those obtained in a conscious child.

In all our cases the airway has been good and in no instance has cyanosis occurred. With the small Magill tube the total cross section of rubber obstructing the airway at the narrowest point-the larynx -is small. The tube causes little trauma in its passage and in addition to its main function provides a good airway should aspiration prove necessary.

Although it is possible to obtain bilateral bronchograms simultaneously, some children will develop cyanosis and anoxia; this is partly due to obstruction of many terminal bronchioles with the medium, and partly to the increased secretions that occur during anaesthesia. The fact that we have avoided carrying out bilateral bronchography at the same time probably explains to some extent why we have not experienced cyanosis and anoxia with this technique.

We feel that this method is reliable and safe. It causes minimal trauma, both physical and mental, to the child. It has given us $95 \%$ of good bronchograms. 